
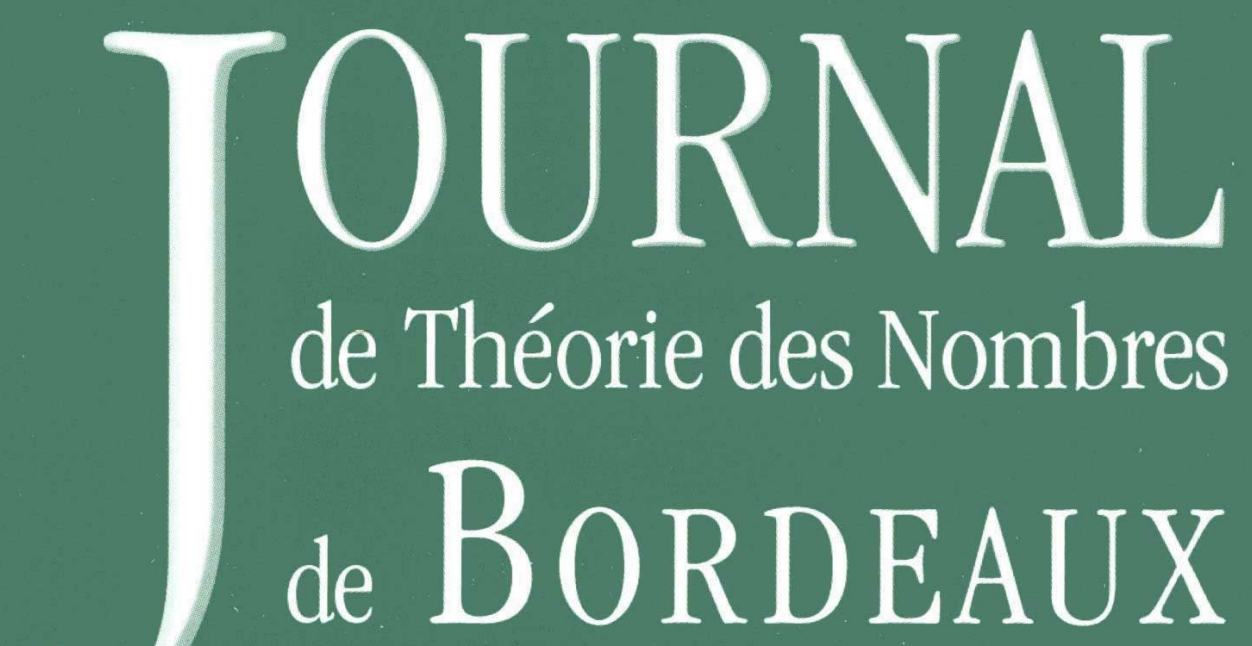

anciennement Séminaire de Théorie des Nombres de Bordeaux

Sandro BETTIN et Sary DRAPPEAU

\title{
Partial sums of the cotangent function
}

Tome 32, no 1 (2020), p. 217-230.

<http://jtnb.centre-mersenne.org/item?id=JTNB_2020__32_1_217_0>

(C) Société Arithmétique de Bordeaux, 2020, tous droits réservés. L'accès aux articles de la revue « Journal de Théorie des Nombres de Bordeaux » (http://jtnb.centre-mersenne.org/), implique l'accord avec les conditions générales d'utilisation (http://jtnb. centre-mersenne.org/legal/). Toute reproduction en tout ou partie de cet article sous quelque forme que ce soit pour tout usage autre que l'utilisation à fin strictement personnelle du copiste est constitutive d'une infraction pénale. Toute copie ou impression de ce fichier doit contenir la présente mention de copyright.

\section{cedram}

Article mis en ligne dans le cadre du

Centre de diffusion des revues académiques de mathématiques

http://www.centre-mersenne.org/ 


\title{
Partial sums of the cotangent function
}

\author{
par SANDRO BETTIN et SARY DRAPPEAU
}

RÉsumÉ. Nous prouvons l'existence de formules de réciprocité pour des sommes de la forme $\sum_{m=1}^{k-1} f\left(\frac{m}{k}\right) \cot \left(\pi \frac{m h}{k}\right)$, où $f$ est une fonction $C^{1}$ par morceaux, qui met en évidence un phénomène d'alternance qui n'apparaît pas dans le cas classique où $f(x)=x$. Nous déduisons des majorations de ces sommes en termes du développement en fraction continue de $h / k$.

Abstract. We prove the existence of reciprocity formulae for sums of the form $\sum_{m=1}^{k-1} f\left(\frac{m}{k}\right) \cot \left(\pi \frac{m h}{k}\right)$ where $f$ is a piecewise $C^{1}$ function, featuring an alternating phenomenon not visible in the classical case where $f(x)=x$. We deduce bounds for these sums in terms of the continued fraction expansion of $h / k$.

\section{1. introduction}

There are several results in the literature proving reciprocity formulae for certain averages of the cotangent function. A prototypical example is the classical Dedekind sum which can be defined as

$$
s\left(\frac{h}{k}\right):=-\frac{1}{4 k} \sum_{m=1}^{k-1} \cot \left(\pi \frac{m}{k}\right) \cot \left(\pi \frac{m h}{k}\right), \quad h, k \in \mathbb{N},(h, k)=1,
$$

and satisfies the well known reciprocity formula

$$
s\left(\frac{h}{k}\right)+s\left(\frac{k}{h}\right)-\frac{1}{12 h k}=\frac{1}{12}\left(\frac{h}{k}+\frac{k}{h}-3\right) .
$$

The Dedekind function has been generalized in several ways, all satisfying some sort of reciprocity (see e.g. [4, 5, 17]).

Another related example is given by the Vasyunin sum

$$
V\left(\frac{h}{k}\right):=\sum_{m=1}^{k-1} \frac{m}{k} \cot \left(\pi \frac{m \bar{h}}{k}\right), \quad h, k \in \mathbb{N},(h, k)=1,
$$

Manuscrit reçu le 6 juin 2019, accepté le 4 juillet 2019

2020 Mathematics Subject Classification. 11L03, 11A55, 11M35.

Mots-clefs. Cotangent sum, continued fraction.

This paper was partially written during a visit of S. Bettin at the Aix-Marseille University and a visit of S. Drappeau at the University of Genova. The authors thank both Institution for the hospitality and Aix-Marseille University and INdAM for the financial support for these visits. S. Bettin is member of the INdAM group GNAMPA and his work is partially supported by PRIN 2015 "Number Theory and Arithmetic Geometry". 
where, here and in the following, the overline indicates any multiplicative inverse modulo the denominator. The Vasyunin sum satisfies the reciprocity formula

$$
\begin{aligned}
V\left(\frac{h}{k}\right)+V\left(\frac{k}{h}\right)=\frac{\log 2 \pi}{\pi}(k+h)+\frac{k-h}{\pi} \log \frac{h}{k} \\
\quad-\frac{\sqrt{h k}}{\pi^{2}} \int_{-\infty}^{\infty}\left|\zeta\left(\frac{1}{2}+i t\right)\right|^{2}\left(\frac{h}{k}\right)^{i t} \frac{\mathrm{d} t}{\frac{1}{4}+t^{2}},
\end{aligned}
$$

where $\zeta(s)$ is the Riemann zeta-function, as well as another one relating $V(\bar{h} / k)$ with $V(\bar{k} / h)$. For this, other generalizations, and the relation of $V$ with the Báez-Duarte and Nyman-Beurling criterion for the Riemann hypothesis we refer to $[2,3,7,8,16]$.

Following the Euclid algorithm and repeatedly applying these results, one can then obtain bounds and asymptotic formulae for these sums in terms of the continued fraction expansion of $h / k$. See, for example [6] and [12].

All of the results mentioned above involve sums over all (non-zero) residues. Having in mind an application to the value distribution of Kashaev's knot invariants [9], we are led to the problem of bounding partial sums of the cotangent function. For example, let

$$
C_{\ell}\left(\frac{h}{k}\right):=\frac{1}{k} \sum_{1 \leq m \leq \ell} \cot \left(\pi \frac{m h}{k}\right), \quad h \in \mathbb{Z}, k \in \mathbb{N},(h, k)=1,1 \leq \ell<k .
$$

Despite the simplicity of this definition, this partial average behave rather erratically, and there is little reason to suspect at first the existence of pure reciprocity formulae such as (1.1).
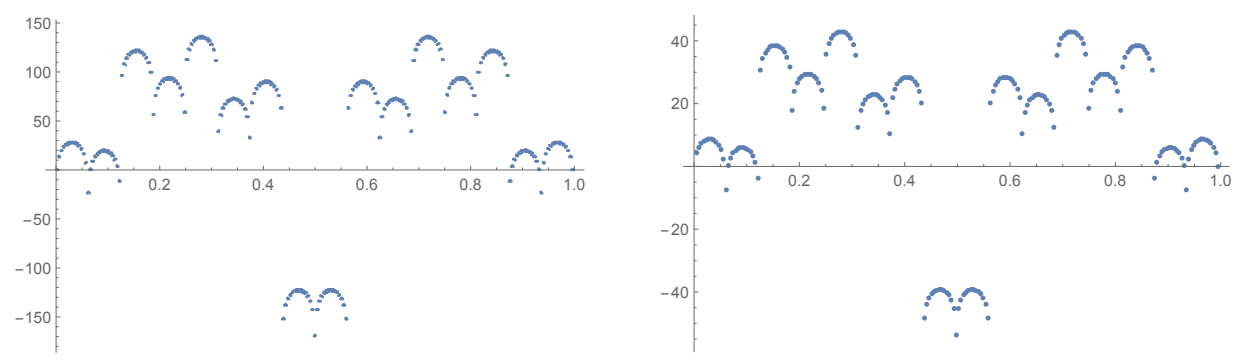

Figure 1.1. The graphs of $\left(\frac{\ell}{677}, C_{\ell}\left(\frac{231}{677}\right)\right)$ as $1 \leq \ell<677$ and of $\left(\frac{\ell}{215}, C_{\ell}\left(\frac{16}{215}\right)\right.$ as $1 \leq \ell<215$.

Figure 1.1 shows the evolution of $C_{\ell}(231 / 677)$ and $C_{\ell}(16 / 215)$ as $\ell$ varies. The continued fraction expansions of $\frac{231}{677}$ and $\frac{16}{215}$ are $\{0 ; 2,1,13,2,3,2\}$ and $\{0 ; 13,2,3,2\}$ respectively, so that the similarity of the two graphs suggests that a reciprocity formula is in action. As we will see below, in this case, the reciprocity formula which we naturally obtain doesn't directly relate 
$C_{\ell}(h / k)$ with $C_{\ell}(k / h)$, nor $C_{\ell}(h / k)$ with $C_{\ell}(\bar{k} / h)$, but rather $C_{\ell}(\bar{h} / k)$ with $C_{\ell^{\prime}}\left(\bar{h} / k_{1}\right)$, where $k_{1} \equiv k_{1}(\bmod h)$ and $0<k_{1} \leq h$, that is, the double iteration of a standard reciprocity formula. This seems to be the first observed instance of such an alternating behaviour in these objects. We give this (non-exact) reciprocity formula in Corollary 10 below.

In general we will consider this question for

$$
S_{f}\left(\frac{h}{k}\right):=\frac{1}{k} \sum_{m=1}^{k-1} f\left(\frac{m}{k}\right) \cot \left(\pi \frac{m h}{k}\right), \quad h \in \mathbb{Z}, k \in \mathbb{N},(h, k)=1 .
$$

where $f$ is a piecewise $\mathcal{C}^{1}$ function. It will follow from our arguments that for generic functions $f, S_{f}(h / k)$ doesn't satisfy a simple reciprocity formula unless $f$ is smooth with only one point of discontinuity other than, possibly, 0 . However, we are able to control its size in all cases, as needed for example in our application to the distribution of the Kashaev invariants.

Theorem 1. Let $f: \mathbb{R} \rightarrow \mathbb{R}$ be a 1-periodic function which is piecewise $\mathcal{C}^{1}$, and continuous except possibly at d points. Let

$$
D_{0}=\max _{x \in \mathbb{R}} \lim _{\varepsilon \rightarrow 0}|f(x+\varepsilon)-f(x-\varepsilon)| \quad \text { and } \quad D_{1}:=\left\|f^{\prime}\right\|_{2} .
$$

Then

$$
\begin{aligned}
& \left|S_{f}\left(\frac{h}{k}\right)\right| \leq \frac{d D_{0}}{\pi k} \sum_{m=1}^{r} v_{m} \log \left(\frac{v_{m}}{v_{m-1}}\right)+O\left(d D_{0}+D_{1}\right), \\
& \left|S_{f}\left(\frac{\bar{h}}{k}\right)\right| \leq \frac{d D_{0}}{\pi} \sum_{m=0}^{r-1} \frac{\log \left(\frac{v_{m+1}}{v_{m}}\right)}{v_{m}}+O\left(d D_{0}+D_{1}\right),
\end{aligned}
$$

where $v_{0}, \ldots, v_{m}$ are the partial quotients of the continued fraction expansion of $h / k$.

Remark 2. Following the approach of [6], it would be possible to compute the distribution of $S_{f}(h / k)$ as $1 \leq h<k$ and $k \rightarrow \infty$ (the method of [14] might also be used with some effort). In particular, one can prove that there is a function $\varepsilon: \mathbb{R}_{>0} \rightarrow \mathbb{R}_{>0}$ with $\lim _{A \rightarrow \infty} \varepsilon(A)=0$, so that for each $A>0$, the number of $h \in[1, k]$ with $(h, k)=1$ and $\left|S_{f}(h / k)\right| \leq A$ is at least $k(1-\varepsilon(A))+o(k)$ as $k \rightarrow \infty$.

Remark 3. If $\{x\}$ denotes the fractional part of $x$, then $\pi \cot (\pi x)-\{x\}^{-1}-$ $\{-x\}^{-1}$ extends to an odd bounded function on $\mathbb{R}$. In particular, Theorem 1 holds also if one replaces $\cot (\pi y)$ by $\left(\{y\}^{-1}-\{y\}^{-1}\right) / \pi$ in the definition of $S_{f}(h / k)$ (or any other 1-periodic function of $y$ having a similar asymptotic behaviour around 0 ).

Theorem 1 is a crucial ingredient in the following law of large numbers for values of the Kashaev invariants of the figure eight knot, which we prove in $[9]$ : 
Theorem 4. For $x \in \mathbb{Q}$, let

$$
J(x):=\sum_{n=0}^{\infty} \prod_{r=1}^{n}\left|1-\mathrm{e}^{2 \pi i r x}\right|^{2}
$$

be the Kashaev invariant of the $4_{1}$ knot. For some constant $\mu>0$, as $Q \rightarrow$ $+\infty$, we have

$$
\log J(x) \sim \mu(\log Q) \log \log Q
$$

for a proportion $1-o(1)$ of fractions $x \in(0,1] \cap \mathbb{Q}$ of reduced denominator at most $Q$.

\section{A reciprocity formula}

2.1. Lemmas. We introduce some notation and give some basic results on the Hurwitz and periodic zeta-functions (cf. [1, Chapter 12.9]). For $x \in \mathbb{R}$ and $\Re(s)>1$, let

$$
\zeta(s, x):=\sum_{n+x>0} \frac{1}{(n+x)^{s}}, \quad F(s, x):=\sum_{n \geq 1} \frac{\mathrm{e}(n x)}{n^{s}}
$$

where $\mathrm{e}(x):=e^{2 \pi i x}$. Notice that we have "periodized" the Hurwitz zetafunction. It is well known that $\zeta(s, x)$ and $F(s, x)$ extend as meromorphic functions on $\mathbb{C}$ and satisfy functional equations. The functional equations are nicely expressed in terms of $\zeta^{ \pm}(s, x):=\zeta(s, x) \pm \zeta(s,-x)$ and $F^{ \pm}(s, x)=$ $F(s, x) \pm F(s,-x)$. Indeed, they become

$$
F^{+}(s, x)=\chi(s) \zeta^{+}(1-s, x), \quad F^{-}(s, x)=2 i \frac{\Gamma(1-s)}{(2 \pi)^{1-s}} \cos \left(\frac{\pi s}{2}\right) \zeta^{-}(1-s, x) .
$$

where $\chi(s):=2 \frac{\Gamma(1-s)}{(2 \pi)^{1-s}} \sin \left(\frac{\pi s}{2}\right)$ is as in the functional equation of the Riemann zeta-function.

We also recall the special values

$$
\begin{aligned}
F^{+}(1, x) & =-\log \left(\left(1-e^{-2 \pi i x}\right)\left(1-e^{2 \pi i x}\right)\right) \\
& =-\log \left(4 \sin (\pi x)^{2}\right), \quad x \notin \mathbb{Z}, \\
F^{-}(1, x) & =2 \pi i \sum_{n \in \mathbb{N}} \frac{\sin (2 \pi n x)}{\pi n}=2 \pi i((x))
\end{aligned}
$$

where $((x)):=0$ if $x \in \mathbb{Z}$ and $((x)):=\frac{1}{2}-\{x\}$ otherwise.

Also, we have the expansion at $s=1$

$$
\zeta(s, x)=\frac{1}{s-1}-\psi(\{x\})+O(s-1),
$$


where $\psi$ is the digamma function [10, Section 8.36], and so also

$$
\begin{array}{r}
F^{+}(1-s, x)=-1-\left(\gamma+\log 2 \pi+\frac{1}{2} \psi(\{x\})+\frac{1}{2} \psi(\{-x\})\right)(1-s) \\
+O\left((s-1)^{2}\right) .
\end{array}
$$

For $h, \ell \in \mathbb{Z}, k \in \mathbb{N}$ we define

$$
\begin{aligned}
V_{1}\left(\frac{h}{k}, \frac{\ell}{k}\right):=2 \sum_{m \geq 1} \sum_{n \geq 1} \frac{\sin \left(2 \pi n m \frac{h}{k}\right) \cos \left(2 \pi m \frac{\ell}{k}\right)}{\pi n m} & \\
& =2 \sum_{m \geq 1} \frac{\cos \left(2 \pi m \frac{\ell}{k}\right)}{m}\left(\left(\frac{m h}{k}\right)\right)
\end{aligned}
$$

which clearly converges since $\sum_{m=1}^{k} \cos (2 \pi m \ell / k)((m h / k))=0$ by parity. Actually, the above computation together with the functional equations for $\zeta(s, x)$ can be used to show that one can smoothly truncate the sum over $m, n$ at height $\ll X k^{1+\varepsilon}$, for any $X \geq 1$, at the cost of an error which is $O\left((X k)^{-100}\right)$, as is done e.g. in [6, p. 11423] with an analogous series.

For $h, p \in \mathbb{Z}, k, q \in \mathbb{N},(h, k)=1$ we also define

$$
V_{2}\left(\frac{h}{k}, \frac{p}{q}\right):=\sum_{\substack{m \in \mathbb{Z} \\\left|m+\frac{p}{q}\right| \geq 1}} \sum_{n \geq 1} \frac{\sin \left(2 \pi \frac{h}{k} n\left|m+\frac{p}{q}\right|\right)}{\pi n\left|m+\frac{p}{q}\right|}=\sum_{\substack{m \in \mathbb{Z} \\\left|m+\frac{p}{q}\right| \geq 1}} \frac{\left(\left(\frac{h}{k}\left|m+\frac{p}{q}\right|\right)\right)}{\left|m+\frac{p}{q}\right|}
$$

where the outer sum is computed by summing together the terms $m$ and $-m$. Again, one easily sees that the series converges and that one can smoothly truncate the sums at $m, n \ll X(q k)^{1+\varepsilon}$ at a cost of an error which is $O\left((X q k)^{-100}\right)$. The manipulation of conditionally convergent sums and integrals in the following is justified by these considerations.

We remark (cf. e.g. Lemma 9 below with $f(x)=x$ for $0<x<1$ ) that $V_{1}\left(\frac{h}{k}, \frac{\ell}{k}\right)$ and $V_{2}\left(\frac{h}{k}, \frac{p}{q}\right)$ reduce to $-\frac{\pi}{k} V\left(\frac{h}{k}\right)$ if $k \mid \ell$ and $p=0$.

We will prove a reciprocity formula relating $V_{1}$ with $V_{2}$, generalizing (1.1), following the same approach as in the proof of [7, Theorem 5]. We shall need the following uniform convexity bound for $F^{+}\left(\frac{1}{2}+i t, x\right)$.

Lemma 5. Let $x \notin \mathbb{Z}$ and

$$
K^{+}(s, x):=F^{+}(s, x)-\chi(s)\left(\{x\}^{s-1}+\{-x\}^{s-1}\right) .
$$

Then $K^{+}\left(\frac{1}{2}+i t, x\right) \ll_{\varepsilon}(1+|t|)^{\frac{1}{4}+\varepsilon}$ uniformly in $x$.

Proof. For $\Re(s)=1+\varepsilon$ we have $K^{+}(s, x) \ll_{\varepsilon} 1$, whereas on $\Re(s)=-\varepsilon$ after applying the functional equation and expanding the resulting series we have $K^{+}(s, x) \ll_{\varepsilon}(1+|s|)^{\frac{1}{2}+\varepsilon}$ uniformly in $x$. Phragmen-Lindelöf's theorem [15, Section 5.61] then gives the claimed bound. 


\subsection{Main reciprocity formula.}

Proposition 6. Let $\ell, h, k \geq 1$ and $(h, k)=1$. For $h \neq 1$, let $\beta:=$ $\left\{\frac{k}{h}\right\}^{-1}\left\{\frac{\ell}{h}\right\}$ if $k \nmid \ell$ and $\beta=0$ if $k \mid \ell$. Then, we have

$$
\begin{aligned}
\frac{1}{h} V_{1}\left(\frac{h}{k}, \frac{\ell}{k}\right)+\delta_{h \neq 1} \frac{1}{k} V_{2}( & \left.\left\{\frac{k}{h}\right\}, \beta\right) \\
& =\left(\frac{\gamma_{h, k, \ell}}{h}-\frac{1}{k}\right) \log \left(\frac{k}{h}\right)+O\left(\frac{1}{k}+\frac{1}{h}\right),
\end{aligned}
$$

where $\delta_{h \neq 1}=0$ if $h=1$ and $\delta_{h \neq 1}=1$ otherwise, and where $\gamma_{h, k, \ell}=1$ if $k \mid \ell$ and otherwise $\gamma_{h, k, \ell}=2$ if $k \leq h$ and $0 \leq \gamma_{h, k, \ell} \leq 1$ if $k>h$.

Remark 7 . For $k \nmid \ell$, the term $\gamma_{h, k, \ell} \log \left(\frac{k}{h}\right) / h$ can be replaced in this estimate by

$$
\frac{1}{h}\left(\log \left\{\frac{\ell}{k}\right\}+\log \left\{-\frac{\ell}{k}\right\}-\log ^{-}\left(\left\{\frac{\ell}{k}\right\} \frac{k}{h}\right)-\log ^{-}\left(\left\{-\frac{\ell}{k}\right\} \frac{k}{h}\right)\right),
$$

where $\log ^{-}(x):=\min (\log x, 0)$.

Proof. We assume $k \nmid \ell$, since otherwise the formula is an immediate consequence of the usual Vasyunin's formula (1.1). Let

$$
I(h, k):=\frac{1}{2 \pi i} \int_{\left(\frac{1}{2}\right)} \frac{F^{+}\left(s, \frac{\ell}{k}\right) \zeta(1-s)}{h^{s} k^{1-s}} \frac{\mathrm{d} s}{s(1-s)},
$$

where $\int_{(c)} \cdot \mathrm{d} s:=\int_{c-i \infty}^{c+i \infty} \cdot \mathrm{d} s$. We will now proceed to evaluate $I(h, k)$ in two different ways.

Throughout, we denote

$$
\alpha=\frac{\ell}{k} .
$$

In the notation of Lemma 5 , we have

$$
\begin{aligned}
I(h, k)=\frac{1}{2 \pi i} \int_{\left(\frac{1}{2}\right)} & \frac{K^{+}(s, \alpha) \zeta(1-s)}{h^{s} k^{1-s}} \frac{\mathrm{d} s}{s(1-s)} \\
& +\frac{1}{2 \pi i} \int_{\left(\frac{1}{2}\right)} \frac{\chi(s)\left(\{\alpha\}^{s-1}+\{-\alpha\}^{s-1}\right) \zeta(1-s)}{h^{s} k^{1-s}} \frac{\mathrm{d} s}{s(1-s)} .
\end{aligned}
$$

By Lemma 5 , the first integral is bounded by

$$
\ll \frac{1}{\sqrt{h k}} \int_{\mathbb{R}} \frac{\left|\zeta\left(\frac{1}{2}+i t\right)\right| \mathrm{d} t}{(1+|t|)^{\frac{3}{2}}} \ll \frac{1}{\sqrt{h k}},
$$


whereas the contribution of $\{\alpha\}$ to the second integral is

$$
\begin{aligned}
\frac{1}{2 \pi i} \int_{\left(\frac{1}{2}\right)} \frac{\chi(s)\{\alpha\}^{s-1} \zeta(1-s)}{h^{s} k^{1-s}} & \frac{\mathrm{d} s}{s(1-s)} \\
& =\frac{1}{k\{\alpha\}} \frac{1}{2 \pi i} \int_{\left(\frac{1}{2}\right)} \zeta(s)\left(\{\alpha\} \frac{k}{h}\right)^{s} \frac{\mathrm{d} s}{s(1-s)} .
\end{aligned}
$$

If $\{\alpha\} \frac{k}{h}<1$ we move the line of integration to $\Re(s)=+\infty$ obtaining a contribution from the double pole at $s=1$ of

$$
\frac{\log \left(\{\alpha\} \frac{k}{h}\right)+\gamma-1}{h} .
$$

If $\{\alpha\} \frac{k}{h} \geq 1$ then we move the integral to $\Re(s)=-\frac{1}{4}$ passing through a simple pole at $s=0$. The contribution of the residue is $-\frac{1}{2 k\{\alpha\}}=O(1 / h)$, whereas the integral on the new line contributes $O\left(\frac{1}{k\{\alpha\}}\left(\{\alpha\} \frac{k}{h}\right)^{-1 / 4}\right)=$ $O(1 / h)$ since $\zeta(-1 / 4+i t) \ll(1+|t|)^{\frac{3}{4}}$. Thus, in both cases we find that the contribution of $\{\alpha\}$ to the second integral is

$$
\frac{\log ^{-}(\{\alpha\} k / h)+O(1)}{h} .
$$

Repeating the same computation for $\{-\alpha\}$ we then obtain our first expression for $I(h, k)$ :

$$
I(h, k)=\frac{\log ^{-}(\{\alpha\} k / h)+\log ^{-}(\{-\alpha\} k / h)+O(1)}{h}+O\left(\frac{1}{\sqrt{k h}}\right) .
$$

Now we compute $I(h, k)$ in a second way. We split the integral into

$$
\begin{aligned}
& \frac{1}{2 \pi i} \int_{\left(\frac{1}{2}\right)} \frac{F^{+}(s, \alpha) \zeta(1-s)}{h^{s} k^{1-s}} \frac{\mathrm{d} s}{s(1-s)} \\
& =\frac{1}{2 \pi i} \int_{\left(\frac{1}{2}\right)} \frac{F^{+}(s, \alpha) \zeta(1-s)}{h^{s} k^{1-s}} \frac{\mathrm{d} s}{1-s}+\frac{1}{2 \pi i} \int_{\left(\frac{1}{2}\right)} \frac{F^{+}(1-s, \alpha) \zeta(s)}{h^{1-s} k^{s}} \frac{\mathrm{d} s}{1-s} \\
& =I_{1}+I_{2},
\end{aligned}
$$

say, where in the second integral we made the change of variables $s \rightarrow 1-s$. We now consider $I_{1}$. We move the line of integration to $\Re(s)=5 / 4$ passing through a simple pole at $s=1$. We obtain

$$
I_{1}=-\frac{F^{+}(1, \alpha)}{2 h}+\frac{1}{2 \pi i} \int_{(5 / 4)} \frac{F^{+}(s, \alpha) \zeta(1-s)}{h^{s} k^{1-s}} \frac{\mathrm{d} s}{1-s} .
$$

By (2.1),

$$
F^{+}(1, \alpha)=-\log \left(4 \sin (\pi \alpha)^{2}\right)=-2 \log \{\alpha\}-2 \log \{-\alpha\}+O(1) .
$$


Also,

$$
\begin{aligned}
& \frac{1}{2 \pi i} \int_{(5 / 4)} \frac{F^{+}(s, \alpha) \zeta(1-s)}{h^{s} k^{1-s}} \frac{\mathrm{d} s}{1-s} \\
& \quad=\sum_{m \in \mathbb{Z}_{\neq 0}} \sum_{n \geq 1} \frac{\mathrm{e}(m \alpha)}{k} \frac{1}{2 \pi i} \int_{(5 / 4)} \chi(1-s)(h n|m| / k)^{-s} \frac{\mathrm{d} s}{1-s} .
\end{aligned}
$$

By the Mellin formula

$$
\frac{1}{2 \pi i} \int_{(5 / 4)} \chi(1-s) u^{-s} \frac{\mathrm{d} s}{1-s}=\frac{\sin (2 \pi u)}{\pi u}
$$

we find

$$
\begin{aligned}
& \frac{1}{2 \pi i} \int_{(2)} \frac{F^{+}(s, \alpha) \zeta(1-s)}{h^{s} k^{1-s}} \frac{\mathrm{d} s}{1-s} \\
&=\sum_{n \geq 1, m \in \mathbb{Z}_{\neq 0}} \frac{\sin (2 \pi h n|m| / k) \mathrm{e}(m \alpha)}{\pi h n|m|}=\frac{1}{h} V_{1}\left(\frac{h}{k}, \alpha\right) .
\end{aligned}
$$

Thus,

$$
I_{1}=\frac{\log \{\alpha\}+\log \{-\alpha\}+O(1)}{h}+\frac{1}{h} V_{1}\left(\frac{h}{k}, \alpha\right) .
$$

As for $I_{2}$ we move the line integration to $\Re(s)=5 / 4$ passing through a double pole at $s=1$. By $(2.2)$ the pole contributes a residue

$$
\begin{aligned}
\frac{\psi(\{\alpha\})+\psi(\{-\alpha\})+2 \log (2 \pi k / h)}{2 k} & \\
= & \frac{-\{\alpha\}^{-1}-\{-\alpha\}^{-1}+2 \log (k / h)+O(1)}{2 k}
\end{aligned}
$$

since $\psi(x)=\frac{1}{x}+O(1)$ for $0<x<1$. The contribution of the integral to $I_{2}$ is

$$
\begin{aligned}
\frac{1}{2 \pi i} \int_{(5 / 4)} \frac{F^{+}(1-s, \alpha) \zeta(s)}{h^{1-s} k^{s}} \frac{\mathrm{d} s}{1-s} \\
=\sum_{m \in \mathbb{Z}} \sum_{n \geq 1} \frac{1}{2 \pi i h} \int_{(5 / 4)} \chi(1-s)\left(\frac{k n}{h}|m+\alpha|\right)^{-s} \frac{\mathrm{d} s}{1-s} \\
=\sum_{m \in \mathbb{Z}} \sum_{n \geq 1} \frac{\sin \left(2 \pi \frac{k}{h} n|m+\alpha|\right)}{\pi k n|m+\alpha|} \\
=\frac{1}{k} \sum_{m \in \mathbb{Z}} \frac{\left(\left(\frac{k}{h}|m+\alpha|\right)\right)}{|m+\alpha|}
\end{aligned}
$$


where in the first line we have applied the functional equation to $F^{+}$and expanded the Dirichlet series.

Now, we observe that the series sums to zero if $h=1$ (since $k|m+\alpha| \in \mathbb{Z}$ in this case) and the claimed result easily follows. Thus, assume $h \neq 1$. We isolate the terms $m \in\{-\lfloor\alpha\rfloor,-\lfloor\alpha\rfloor-1,\lfloor-\beta\rfloor,\lfloor-\beta\rfloor+1\}$ from the sum, where

$$
\beta:=\left\{\frac{k}{h}\right\}^{-1}\left\{\frac{\ell}{h}\right\} .
$$

The terms $m \in\{-\lfloor\alpha\rfloor,-\lfloor\alpha\rfloor-1\}$ contribute

$$
\frac{\left(\left(\frac{k}{h}\{\alpha\}\right)\right)}{k\{\alpha\}}+\frac{\left(\left(\frac{k}{h}\{-\alpha\}\right)\right)}{k\{-\alpha\}}
$$

whereas the contribution of $m^{\prime} \in\{\lfloor-\beta\rfloor,\lfloor-\beta\rfloor+1\}$ (with $m^{\prime} \neq-\lfloor\alpha\rfloor$, $-\lfloor\alpha\rfloor-1)$ is bounded by

$$
\frac{1}{k} \frac{\left(\left(\frac{k}{h}\left|m^{\prime}+\alpha\right|\right)\right)}{\left|m^{\prime}+\alpha\right|} \ll \frac{1}{k} .
$$

Next, we replace $\alpha$ in the denominator by $\beta$. The error in doing so is

$$
\frac{1}{k} \sum_{m \neq-\lfloor\alpha\rfloor,-\lfloor\alpha\rfloor-1,\lfloor-\beta\rfloor,\lfloor-\beta\rfloor+1}\left(\left(\frac{k}{h}|m+\alpha|\right)\right)\left(\frac{1}{|m+\{\alpha\}|}-\frac{1}{|m+\beta|}\right) \ll \frac{1}{k} .
$$

We can then include again the terms $-\lfloor\alpha\rfloor$ and $-\lfloor\alpha\rfloor-1$ (when they are different from $\lfloor-\beta\rfloor,\lfloor-\beta\rfloor+1)$ at the cost of an error which is analogously seen to be $O(1 / k)$, obtaining

$$
\begin{aligned}
& \frac{1}{k} \sum_{m \in \mathbb{Z}} \frac{\left(\left(\frac{k}{h}|m+\alpha|\right)\right)}{|m+\alpha|} \\
& =\frac{1}{k} \sum_{\substack{m \in \mathbb{Z}, m \neq\lfloor-\beta\rfloor,\lfloor-\beta\rfloor+1}} \frac{\left(\left(\frac{k}{h}|m+\alpha|\right)\right)}{|m+\beta|}+\frac{\left(\left(\frac{k}{h}\{\alpha\}\right)\right)}{k\{\alpha\}}+\frac{\left(\left(\frac{k}{h}\{-\alpha\}\right)\right)}{k\{-\alpha\}}+O\left(\frac{1}{k}\right) .
\end{aligned}
$$

By periodicity of the sine we then have

$$
\begin{aligned}
& \frac{1}{k} \sum_{\substack{m \in \mathbb{Z}, m \neq\lfloor-\beta\rfloor,\lfloor-\beta\rfloor+1}} \frac{\left(\left(\frac{k}{h}|m+\alpha|\right)\right)}{|m+\beta|} \\
& =\frac{1}{k} \sum_{\substack{m \in \mathbb{Z}, m \neq\lfloor-\beta\rfloor,\lfloor-\beta\rfloor+1}} \frac{\left(\left(\frac{k}{h}|m+\beta|\right)\right)}{|m+\beta|}=\frac{1}{k} V_{2}\left(\left\{\frac{k}{h}\right\}, \beta\right)+O\left(\frac{1}{k}\right) .
\end{aligned}
$$


Finally, we observe that

$$
\begin{aligned}
& \frac{\left(\left(\frac{k}{h}\{\alpha\}\right)\right)}{k\{\alpha\}}+\frac{\left(\left(\frac{k}{h}\{-\alpha\}\right)\right)}{k\{-\alpha\}} \\
& \quad=\frac{1}{2 k\{\alpha\}}+\frac{1}{2 k\{-\alpha\}}-\frac{\left\{\frac{k}{h}\{\alpha\}\right\}}{k\{\alpha\}}-\frac{\left\{\frac{k}{h}\{-\alpha\}\right\}}{k\{-\alpha\}}+O\left(\frac{1}{h}\right) \\
& =\frac{1}{2 k\{\alpha\}}+\frac{1}{2 k\{-\alpha\}}+O\left(\frac{1}{h}\right) .
\end{aligned}
$$

Indeed, the third term is $O(1 / h)$ (and similarly for the fourth) since, if $0 \leq$ $\ell^{\prime} \leq k$ and $0 \leq \ell^{\prime \prime} \leq h$ are such that $\ell^{\prime} \equiv \ell(\bmod k)$ and $\ell^{\prime \prime} \equiv \ell^{\prime}(\bmod h)$, then $\left\{\frac{k}{h}\{\alpha\}\right\} / k\{\alpha\}=\ell^{\prime \prime} /\left(\ell^{\prime} h\right) \leq \frac{1}{h}$.

By the above computations we then have

$$
\begin{aligned}
& \frac{1}{2 \pi i} \int_{(5 / 4)} \frac{F^{+}(1-s, \alpha) \zeta(s)}{h^{1-s} k^{s}} \frac{\mathrm{d} s}{1-s} \\
& \quad=\frac{1}{k} V_{2}\left(\left\{\frac{k}{h}\right\}, \beta\right)+\frac{1}{2 k\{\alpha\}}+\frac{1}{2 k\{-\alpha\}}+O\left(\frac{1}{k}+\frac{1}{h}\right)
\end{aligned}
$$

and so, adding the contribution of the residue (2.6) we have

$$
I_{2}=V_{2}\left(\left\{\frac{k}{h}\right\}, \beta\right) / k+\log (k / h) / k+O\left(\frac{1}{k}+\frac{1}{h}\right) .
$$

Thus,

$$
\begin{aligned}
I(h, k)=I_{1}+I_{2}=\frac{\log \{\alpha\}}{}+\log \{-\alpha\} & +\frac{1}{h} V_{1}\left(\frac{h}{k}, \alpha\right) \\
+ & \frac{1}{k} V_{2}\left(\left\{\frac{k}{h}\right\}, \beta\right)+\frac{\log (k / h)}{k}+O\left(\frac{1}{k}+\frac{1}{h}\right) .
\end{aligned}
$$

Comparing this with (2.5) we obtain the claimed identity, since for $0<x<$ $1, y>0$ we have

$$
\log x+\log (1-x)-\log ^{-}(x y)-\log ^{-}((1-x) y)=-\gamma \log y+O(1)
$$

where $\gamma=2$ if $0<y \leq 1$ and $0<\gamma \leq 1$ otherwise.

2.3. Bound for $V_{1}$ in terms of the continued fraction expansion. We now iterate the relation (2.4) in order to obtain a bound for individual values of $V_{1}$. 
Corollary 8. Let $\ell, h, k \in \mathbb{N}$ with $h<k$ and $(h, k)=1$. Then, uniformly for $\alpha \in \frac{1}{k} \mathbb{Z}$, we have

$$
\begin{aligned}
& \left|V_{1}\left(\frac{h}{k}, \alpha\right)\right| \leq \sum_{m=0}^{r-1} \frac{\log \left(v_{m+1} / v_{m}\right)}{v_{m}}+O(1), \\
& \left|V_{1}\left(\frac{\bar{h}}{k}, \alpha\right)\right| \leq \frac{1}{k} \sum_{m=1}^{r} v_{m} \log \left(v_{m} / v_{m-1}\right)+O(1),
\end{aligned}
$$

where $v_{m}$ is the $m$-th partial quotient of $\frac{h}{k}$.

Proof. By Proposition 6, recalling the notation $\beta:=\left\{\frac{k}{h}\right\}^{-1}\left\{\frac{\ell}{h}\right\}$ if $k \nmid \ell$ and $\beta:=0$ otherwise, we have

$$
\begin{aligned}
& \left|k V_{1}\left(\frac{h}{k}, \frac{\ell}{k}\right)\right| \leq \delta_{h \neq 1}\left|h V_{2}\left(\left\{\frac{k}{h}\right\}, \beta\right)\right|+k \log \left(\frac{k}{h}\right)+O(k) \quad \text { if } h \leq k \\
& \left|h V_{2}\left(\frac{k}{h}, \beta\right)\right| \leq\left|k V_{1}\left(\frac{h}{k}, \frac{\ell}{k}\right)\right|+h \log \left(\frac{h}{k}\right)+O(h) \quad \text { if } h \geq k, h \neq 1
\end{aligned}
$$

Now, let $h / k=\left[0 ; b_{1}, \ldots, b_{r}\right]$ be the continued fraction expansion of $h / k$, with $b_{r} \neq 1$ if $r>1$. Also, let $h^{*} \in[1, k]$ be such that $h^{*} \equiv \overline{(-1)^{r+1} h}(\bmod k)$ (in particular $\left.h^{*} / k=\left[0, b_{r}, \ldots, b_{1}\right]\right)$. The Euclid algorithm on $h^{*}$ and $k$ can be written as (see [13])

$$
\begin{aligned}
& v_{r}=k, \quad v_{r-1}=h^{*}, \\
& v_{\ell+1}=b_{\ell+1} v_{\ell}+v_{\ell-1}, \quad \ell=0, \ldots r .
\end{aligned}
$$

Then, alternating the use of (2.7)-(2.8) and the reduction modulo the denominator in $V_{1}$, we obtain

$$
\begin{aligned}
k\left|V_{1}\left(\frac{\bar{h}}{k}, \alpha\right)\right| \leq \sum_{m=1}^{r}\left(v_{m} \log \left(v_{m} / v_{m-1}\right)+O\left(v_{m}\right)\right) \\
=\sum_{m=1}^{r} v_{m} \log \left(v_{m} / v_{m-1}\right)+O(k),
\end{aligned}
$$

as desired, where the last step follows since $v_{n-2} \leq v_{n} / 2$ for all $n$.

Indicating with $u_{m} / v_{m}$ and $u_{m}^{\prime} / v_{m}^{\prime}$ the $m$-th convergents of $h / k$ and $h^{*} / k$ respectively (with $v_{-1}=v_{-1}^{\prime}=0$ ), one has $k=v_{s} v_{r-s}^{\prime}+v_{s-1} v_{r-s-1}^{\prime}$ for all $0 \leq s \leq r$ (see [11, p. 91-92]). In particular, $k / 2 \leq v_{s} v_{r-s}^{\prime} \leq k$. Thus, by (2.9) we have

$$
\begin{aligned}
k\left|V_{1}\left(\frac{h}{k}, \alpha\right)\right| & \leq \sum_{m=1}^{r} v_{m}^{\prime} \log \left(v_{m}^{\prime} / v_{m-1}^{\prime}\right)+O(k) \\
& \leq k \sum_{m=0}^{r-1} \frac{\log \left(v_{m+1} / v_{m}\right)}{v_{m}}+O(k) .
\end{aligned}
$$




\section{Proof of Theorem 1}

We will deduce Theorem 1 from Corollary 8 and the following lemma, which shows that $S_{f}(h / k)$ is, up to a small error, a linear combination of $V_{1}\left(\frac{\bar{h}}{k}, \frac{\ell_{j}}{k}\right)$ for various values of $\ell_{j}$.

Lemma 9. Let $h \in \mathbb{Z}, k \in \mathbb{N}$ with $(h, k)=1$. Let $f: \mathbb{R} \rightarrow \mathbb{R}$ be a 1 -periodic function which is piecewise $\mathcal{C}^{1}$, and continuous everywhere except possibly at $d$ points $\frac{\ell_{1}}{k}, \ldots, \frac{\ell_{d}}{k}$, with $0 \leq \ell_{1}<\cdots<\ell_{d}<k$, and assume $f$ is left and right differentiable at such points. Also, assume $2 f\left(\frac{\ell_{i}}{k}\right)=f\left(\frac{\ell_{i}}{k}+\right)+f\left(\frac{\ell_{i}}{k}-\right)$, where $f(x \pm):=\lim _{\varepsilon \rightarrow 0^{+}} f(x \pm \varepsilon)$. Then,

$$
S_{f}\left(\frac{h}{k}\right)=\frac{1}{\pi} \sum_{j=1}^{d} V_{1}\left(\frac{\bar{h}}{k}, \frac{\ell_{i}}{k}\right)\left(f\left(\frac{\ell_{j}}{k}+\right)-f\left(\frac{\ell_{j}}{k}-\right)\right)+O\left(\left\|f^{\prime}\right\|_{2}\right) .
$$

Proof. We have the Fourier expansion

$$
f(x)=\sum_{n \in \mathbb{Z}} \widehat{f}(n) \mathrm{e}(-n x), \quad x \in \mathbb{R}
$$

where, for $n \neq 0$,

$$
\widehat{f}(n)=\sum_{j=1}^{d} \frac{f\left(\frac{\ell_{j}}{k}-\right)-f\left(\frac{\ell_{j}}{k}+\right)}{2 \pi i n} \mathrm{e}\left(n \ell_{j} / k\right)-\frac{\widehat{f}^{\prime}(n)}{2 \pi i n},
$$

and $\widehat{f}^{\prime}(n):=\int_{0}^{1} f^{\prime}(y) \mathrm{e}(n y) \mathrm{d} y$. Note that, by the Bessel inequality,

$$
\sum_{n \neq 0}\left|\frac{\hat{f}^{\prime}(n)}{n}\right| \ll\left\|f^{\prime}\right\|_{2}
$$

Now, we have

$$
\sum_{m=1}^{k-1} \cot \left(\pi \frac{m h}{k}\right) \mathrm{e}\left(-\frac{n m}{k}\right)=-2 i k\left(\left(\frac{n \bar{h}}{k}\right)\right)
$$

and thus,

$$
\begin{aligned}
& \sum_{m=1}^{k-1} f\left(\frac{m}{k}\right) \cot \left(\frac{\pi m h}{k}\right) \\
& \quad=-2 i k \sum_{n \in \mathbb{Z}_{\neq 0}}\left(\left(\frac{n \bar{h}}{k}\right)\right)\left(\sum_{j=1}^{d} \frac{f\left(\frac{\ell_{j}}{k}-\right)-f\left(\frac{\ell_{j}}{k}+\right)}{2 \pi i n} \mathrm{e}\left(n \ell_{j} / k\right)-\frac{\widehat{f}^{\prime}(n)}{2 \pi i n}\right) .
\end{aligned}
$$

Grouping this with the definition (2.3) and the bound (3.1) yields our claim. 
Proof of Theorem 1. At the cost of committing an error of size $O\left(d D_{0}\right)$ in $S_{f}(h / k)$, we modify $f$ so that all of its $d$ points of discontinuity are at rationals of the form $\frac{\ell}{k}$ with $2 f\left(\frac{\ell}{k}\right)=f\left(\frac{\ell}{k}+\right)+f\left(\frac{\ell}{k}-\right)$. Theorem 1 then follows by Proposition 1 and Lemma 9 .

Particular case: partial cotangent sums. For the partial cotangent sums, alluded to in the introduction (Figure 1.1), since there is only one point of discontinuity other than 0 , we indeed obtain a (non-exact) reciprocity relation in the following form.

Corollary 10. Let $h \in \mathbb{Z}, k \in \mathbb{N}$ with $(h, k)=1,1 \leq h<k$, and let $0<\ell<k$. Let $k_{1} \equiv k(\bmod h), \ell^{\prime} \equiv \ell(\bmod h)$ with $1 \leq k_{1}, \ell^{\prime} \leq h$ and let $\ell_{1} \equiv \ell^{\prime}\left(\bmod k_{1}\right), h_{1} \equiv h\left(\bmod k_{1}\right)$ with $0 \leq h_{1}<k_{1}, 1 \leq \ell_{1} \leq k_{1}$. Then,

$$
C_{\ell}(\bar{h} / k)-C_{\ell_{1}}\left(\overline{h_{1}} / k_{1}\right)=\frac{1}{\pi}\left(\gamma_{h, k, \ell}-1\right) \log \frac{k}{h}+O\left(\frac{h}{k_{1}}\right) .
$$

with $0 \leq \gamma_{h, k, \ell} \leq 1$.

Proof. By Lemma 9 we have $C_{\ell}(\bar{h} / k)=\frac{1}{\pi}\left(V_{1}\left(\frac{h}{k}, \frac{\ell}{k}\right)-V\left(\frac{h}{k}, 0\right)\right)+O(1)$. The result then follows, since applying Proposition 6 to $\left(\frac{h}{k}, \frac{\ell}{k}\right)$ and to $\left(\frac{h}{k_{1}}, \frac{\ell^{\prime}}{k_{1}}\right) \equiv$ $\left(\frac{h_{1}}{k_{1}}, \frac{\ell_{1}}{k_{1}}\right)(\bmod 1)$ we obtain

$V_{1}\left(\frac{h}{k}, \frac{\ell}{k}\right)-V_{1}\left(\frac{h}{k_{1}}, \frac{\ell^{\prime}}{k_{1}}\right)=\left(\gamma_{h, k, \ell}-\frac{h}{k}\right) \log \frac{k}{h}+\frac{h}{k_{1}} \log \frac{k_{1}}{h}+O\left(\frac{h}{k_{1}}\right)$.

\section{References}

[1] T. M. Apostol, Introduction to analytic number theory, Undergraduate Texts in Mathematics, Springer, 1976.

[2] L. BÁEz-DuARte, "A strengthening of the Nyman-Beurling criterion for the Riemann hypothesis", Atti Accad. Naz. Lincei, Cl. Sci. Fis. Mat. Nat., IX. Ser., Rend. Lincei, Mat. Appl. 14 (2003), no. 1, p. 5-11.

[3] B. BAGCHI, "On Nyman, Beurling and Baez-Duarte's Hilbert space reformulation of the Riemann hypothesis", Proc. Indian Acad. Sci., Math. Sci. 116 (2006), no. 2, p. 137-146.

[4] M. BECK, "Dedekind cotangent sums", Acta Arith. 109 (2003), no. 2, p. 109-130.

[5] B. C. Berndt, "Dedekind sums and a paper of G. H. Hardy", J. Lond. Math. Soc. 13 (1976), no. 1, p. 129-137.

[6] S. Bettin, "On the distribution of a cotangent sum", Int. Math. Res. Not. 2015 (2015), no. 21 , p. 11419-11432.

[7] S. Bettin \& B. Conrey, "Period functions and cotangent sums", Algebra Number Theory 7 (2013), no. 1, p. 215-242.

[8] S. Bettin \& J. B. Conrey, "A reciprocity formula for a cotangent sum", Int. Math. Res. Not. 2013 (2013), no. 24, p. 5709-5726.

[9] S. Bettin \& S. Drappeau, "Modularity and value distribution of quantum invariants of hyperbolic knots", https://arxiv.org/abs/1905.02045v3, 2020.

[10] I. S. Gradshteyn \& I. M. Ryzhik, Table of integrals, series, and products, Elsevier; Academic Press Inc., 2007.

[11] H. Heilbronn, "On the average length of a class of finite continued fractions", in Number Theory and Analysis (Papers in Honor of Edmund Landau), Plenum Press, 1969, p. 87-96.

[12] D. Hickerson, "Continued fractions and density results for Dedekind sums", J. Reine Angew. Math. 290 (1977), p. 113-116. 
[13] A. Y. Khinchin, Continued fractions, P. Noordhoff, 1963, translated by Peter Wynn.

[14] H. MAiER \& M. T. RAssias, "Generalizations of a cotangent sum associated to the Estermann zeta function", Commun. Contemp. Math. 18 (2016), no. 1, article ID 1550078 (89 pages).

[15] E. C. Titchmarsh, The theory of functions, 2nd ed., Oxford University Press, 1939.

[16] V. I. VAsyunin, "On a biorthogonal system associated with the Riemann hypothesis", Algebra Anal. 7 (1995), no. 3, p. 118-135.

[17] D. ZAGier, "Higher dimensional Dedekind sums", Math. Ann. 202 (1973), p. 149-172.

\section{Sandro Bettin}

DIMA - Dipartimento di Matematica

Via Dodecaneso, 35

16146 Genova, Italy

E-mail: bettin@dima.unige.it

\section{Sary Drappeau}

Aix Marseille Université, CNRS

Centrale Marseille, I2M UMR 7373

13453 Marseille, France

E-mail: sary-aurelien.drappeau@univ-amu.fr 